\title{
Declínio da cárie dentária em escolares do município de Rio das Pedras, SP, Brasil
}

\author{
Dental caries decline in scholars from the city of Rio das Pedras, SP, Brazil
}

\section{Lilian Berta Rihs ${ }^{1}$, Renato Pereira da Silva ${ }^{2}$, Karine Laura Cortellazzi ${ }^{3}$, Maria da Luz Rosário de Sousa ${ }^{4}$}

\section{Resumo}

Os objetivos deste trabalho foram avaliar o declínio na prevalência de cárie dentária entre 1998 e 2004 e verificar a desigualdade na distribuição da doença em pré-escolares e escolares no município de Rio das Pedras, SP, Brasil, no mesmo período. A amostra foi composta por 739 crianças de 5 a 12 anos de idade provenientes de escolas públicas e particulares em 1998 e um total de 1159 indivíduos de 5 a 14 anos de escolas públicas foi avaliado em 2004. O Significant Caries Index (SiC) e o Coeficiente de Gini foram aplicados. Verificou-se um aumento de $61,3 \%(p=0,009)$ e $28 \%(p=0,0002)$ de crianças livres de cárie aos 5 e aos 12 anos de idade, respectivamente. Em 1998, a média do índice ceod aos 5 anos de idade foi de 3,8 (IC95\%=2,9-4,7) e de 2,4 (IC95\%=2,0-2,8) em 2004. A média do índice CPOD aos 12 anos de idade foi de 4,8 (IC95\%=4,0-5,6) em 1998 e de 2,7 (IC95\%=2,3-3,1) em 2004. A diminuição do componente cariado, tanto do ceod quanto do CPOD, foi o que mais contribuiu para a queda dos índices de cárie nesta população. A média do SiC, aos 12 anos de idade, diminuiu consideravelmente no período, passando de 9,03, em 1998, a 5,7, em 2004. Em conclusão, apesar da melhoria na saúde bucal da população, verifica-se que ainda existe uma parcela de indivíduos com altos níveis de cárie aos 12 anos e que a desigualdade na distribuição da doença aumentou no período estudado.

Palavras-chave: Saúde bucal, Epidemiologia, Cárie dentária, Escolares, Pré-Escolares

\section{Abstract}

This study aimed to evaluate the decrease in dental caries prevalence between 1998 and 2004 and to verify the disease distribution inequalities in preschool children and schoolchildren from Rio das Pedras, SP, Brazil, in the same period. The sample comprised 739 children aged 5 to 12 years attending public and private schools in 1998 and 1159 subjects aged 5 to 14 years from public schools in 2004. The Significant Caries Index ( $\mathrm{SiC}$ ) and the Gini Coefficient were measured. It was observed an increase of $61.3 \% \quad(p=0.009)$ and $28 \%(p=0.0002)$ of caries free in 5 and 12 years-old children, respectively. The mean dmft for 5 -year-olds was $3.8(\mathrm{Cl}$-95\%=2.9-4.7) in 1998 and $2.4(\mathrm{Cl} 95 \%=2.0-2.8)$ in 2004. The DMFT for 12-yearolds was $4.8(\mathrm{Cl} 95 \%=4.0-5.6)$ in 1998 , and $2.7(\mathrm{Cl} 95 \%=2.3-3.1)$ in 2004. The decrease of the decayed component of dmft and DMFT indexes was the major contributor for decreasing the caries index of the population. The mean "SIC" index for children aged 12 years decreased from 9.03 to 5.7 from 1998 to 2004. In conclusion, although an improvement in oral health could be observed in the population, some subjects aged 12 years has shown high levels of caries lesions and caries inequalities distribution increased in the period of the study.

Keywords: Oral health, Epidemiology, Dental caries, Preschool children, Schoolchildren
${ }^{1}$ Doutora em Odontologia em Saúde Coletiva pela FOP/Unicamp 2 Doutor em Odontologia em Saúde Coletiva pela FOP/Unicamp ${ }^{3}$ Doutora em Odontologia em Saúde Coletiva pela FOP/Unicamp ${ }^{4}$ Professora Titular do Departamento de Odontologia Social pela FOP/Unicamp, Doutora em Saúde Pública - USP

Correspondência: Profa. Dra. Maria da Luz Rosário Sousa Endereço: Departamento de Odontologia Social - Faculdade de Odontologia de Piracicaba, Caixa Postal n52 / Universidade Estadual de Campinas UNICAMP. Av. Limeira, 901 - Areião - CEP 13414-903 - Piracicaba - SP - Brasil

\section{Introdução}

No Brasil, vários estudos direcionados ao conhecimento das condições de saúde bucal da população relatam um declínio na experiência de cárie entre crianças e adolescentes (BRASIL, 1988; TRAEBERT et al., 2001; ANTUNES et al., 2002; BALDANI et al., 2002; CANGUSSU, et al., 2002; CYPRIANO et al., 2003; TAGLIAFERRO et al., 2003, GUSHI et al., 2005).

Entretanto, esse declínio na prevalência da cárie dentária ocorreu acompanhado por um fenômeno conhecido como polarização da doença, que consiste na concentração da maior parte da cárie ou das necessidades de tratamento em uma pequena parcela da população (WEYNE, 1997). Devido a essa mudança no padrão da doença, um novo índice denominado Significant Caries Index (SiC) foi proposto no ano 2000 (BRATTHALL, 2000), a fim de focalizar a atenção para aqueles indivíduos com os mais altos índices de cárie na população investigada.

Além disso, com a queda dos índices de cárie na população e consequente verificação de parcela da população ainda apresentando altos índices da doença, trabalhos na literatura têm discutido a interação de possíveis fatores associados a esse fenômeno. Vários estudos têm demonstrado a influência de fatores sociais e econômicos na determinação da cárie, relacionando o nível socioeconômico à prevalência, bem como à severidade da doença (PERES et al., 2000; TRAEBERT et al., 2001; ANTUNES et al., 2002; BALDANI et al., 2002; CANGUSSU et al., 2002; TINANOFF et al., 2002). Buscando verificar o aumento da desigualdade social na expressão da cárie dentária, Antunes e colaboradores (2002) propuseram o coeficiente de Gini que avalia a desigualdade na distribuição da cárie dentária durante um período de tempo.

Desta forma, os objetivos deste trabalho são verificar a prevalência de cárie dentária em escolares de 5 a 14 anos, em 2004, e descrever o declínio de cárie em escolares de 5 a 12 anos, de 1998 a 2004, no município de Rio das Pedras. Além disso, buscou-se comparar um indicador de severidade de cárie (Significant Caries Index - SiC Index) e desigualdade na distribuição da doença (Coeficiente de Gini) neste mesmo período. 


\section{Materiais e Métodos}

O município de Rio das Pedras está localizado a 154 km da cidade de São Paulo, apresenta uma população de 30.000 (Prefeitura Municipal de Rio das Pedras, 2006) habitantes e o início da fluoretação das águas de abastecimento público se deu em 2001 - comunicação pessoal com o Coordenador de Saúde Bucal do município de Rio das Pedras em 2004. No ano de 1998, a Secretaria Estadual de Saúde do Estado de São Paulo realizou um levantamento epidemiológico em saúde bucal e Rio das Pedras foi um dos municípios sorteados, sendo avaliado o grupo etário de 5 a 12 anos. É importante ressaltar que no ano do levantamento epidemiológico a cidade ainda não contava com flúor nas águas de abastecimento público e não possuía dados prévios de cárie dentária.

Em 2004, um novo levantamento epidemiológico em crianças de 5 a 14 anos foi conduzido pelo próprio município com o intuito de conhecer o perfil de saúde bucal dos pré-escolares e escolares da rede pública de ensino. Neste momento, a cidade de Rio das Pedras já contava com a fluoretação das águas de abastecimento público, que teve início em 2001.

O delineamento seguido nos dois levantamentos, 1998 e 2004, seguiu critérios semelhantes e foram realizados ambos sob coordenação da Faculdade de Odontologia de Piracicaba - UNICAMP.

O tamanho amostral por idade e por município, no ano de 1998, foi calculado conforme recomendação da OMS (WHO, 1997), que considera 40 crianças por idade suficiente para a obtenção de dados confiáveis quando a prevalência de cárie é moderada ou alta. Assim, admitindo-se um erro de desenho de 2, como também $20 \%$ de perda amostral, tem-se: $(40 \times 2)+20 \%=96$ indivíduos por idade (SES/SP, 1999). No ano de 2004, para as idades de 5 e 12 anos de idade, o cálculo do tamanho da amostra foi baseado na experiência prévia de cárie deste município (ceod de 3,8 [DP=3,6] aos 5 anos e CPOD de 4,8 [DP=3,9] aos 12 anos) obtida no estudo realizado em 1998, adotando-se para tal nível de confiança de 95\%, precisão de $20 \%$ e efeito do desenho (deff) de 2 . Somaram-se $20 \%$ a este total, a fim de compensar eventuais perdas e recusas, resultando em um tamanho amostral de 207 crianças para a idade de 5 anos e de 152 aos 12 anos. Para as outras idades, seguiu-se a metodologia adotada no levantamento de 1998.

O sorteio das instituições de ensino, bem como dos participantes, foi realizado segundo recomendações descritas no Manual de Instruções idealizado para o levantamento Estadual de 1998 (SES/SP, 1999). No ano de 2004, o estudo foi realizado segundo recomendações descritas no Manual do Coordenador utilizado no Levantamento Nacional (BRASIL, 2003).

Os códigos utilizados para a avaliação de cárie dentária seguiram as recomendações da Organização Mundial de Saúde (WHO, 1997), adaptados pela FSP/USP (SES/SP, 1999), sendo utilizados os índices ceod e CPOD.

Também utilizou-se, para a análise dos resultados, o Significant Caries Index - SiC Index (BRATTHALL, 2000) que é calculado separando-se do grupo geral um terço dos indivíduos que tenham apresentado os valores mais altos de experiência de cárie. Neste subgrupo, calcula-se o CPOD e se obtém o valor do SiC Index.

O coeficiente de Gini avalia a desigualdade na distribuição da cárie dentária, sendo que o valor pode variar de $0 \mathrm{a}$ 1 , sendo o valor 0 considerado como igualdade total e o valor 1 corresponde à máxima desigualdade. Para o seu cálculo, foi utilizada uma planilha eletrônica disponível no endereço eletrônico $<$ http://www.fo.usp.br/arquivos/Gini_calculation_for_caries_distribution.zip>.

Os exames dos dois levantamentos epidemiológicos foram realizados por dois cirurgiões-dentistas devidamente calibrados, cujo processo de calibração em cada uma das ocasiões efetuou-se em 36 horas divididas entre discussões teóricas e atividades práticas, simulando as diferentes condições e situações que a equipe encontraria durante a realização do trabalho prático.

Na calibração em 1998, a porcentagem de concordância interexaminadores para cárie foi de $97,5 \%$. Para o erro intraexaminador, foi de $98,4 \%$. Em 2004, a concordância interexaminadores foi de $99 \%$, com valor de Kappa de 0,98. Esses resultados são aceitáveis para estudos epidemiológicos em saúde bucal sobre cárie dentária (WHO, 1987), comprovando a confiabilidade dos dados coletados.

Os exames foram realizados em local com luz natural, utilizando-se espelho bucal plano e sonda CPI para levantamentos epidemiológicos (WHO, 1997). Os dados foram registrados por anotadores previamente calibrados, em fichas individuais.

$\mathrm{Na}$ análise estatística, utilizaram-se os testes MannWhitney, Qui-Quadrado com nível de significância de 5\%.

Em se tratando de pesquisas que envolvem seres humanos, os projetos foram submetidos à avaliação do Comitê de Ética em Pesquisa, sendo aprovados em 1998 pelo Comitê de Ética em Pesquisa da Faculdade de Saúde Pública da USP (COEPUSP/62/98), e, em 2004, pelo Comitê de Ética da Faculdade de Odontologia de Piracicaba - UNICAMP (COEP155/2004). Também obteve-se, em ambos os estudos, a autorização das pessoas (ou responsáveis) que participariam do trabalho, através do termo de consentimento livre e esclarecido para pesquisa.

\section{Resultados}

A distribuição da amostra estudada, tanto em 1998 quanto em 2004, pode ser visualizada na Tabela 1. A mesma tabela traz as frequências relativas dos indivíduos livres de cárie (para a dentição mista, considerou-se como indivíduo livre de cárie, os que apresentaram ceod + CPOD $=0$ ) para cada uma das idades estudadas. Em 2004, aos 5 e 12 anos de idade, verificou-se maior percentual de crianças livres de cárie, quando comparados aos escolares do ano de $1998(p=0,009$ e $p=0,0002$, respectivamente).

Tabela 1. Distribuição dos escolares segundo o ano de estudo e em relação aos livres de cárie (ceod=0 e CPOD=0). Rio das Pedras, 1998 e 2004.

\begin{tabular}{|c|c|c|c|c|}
\hline & $\begin{array}{c}\text { Amostra } \mathrm{n} \\
(\%)\end{array}$ & $\begin{array}{l}\text { Livres de } \\
\text { cárie (\%) }\end{array}$ & & \\
\hline Idade & 1998 & 2004 & 1998 & 2004 \\
\hline 5 & $67(9,1)$ & $214(18,5)$ & 28,4 & 46,3 \\
\hline 6 & $98(13,3)$ & $100(8,6)$ & 19,4 & 42,0 \\
\hline 7 & $94(12,7)$ & $101(8,7)$ & 13,8 & 33,7 \\
\hline 8 & $110(14,9)$ & $100(8,6)$ & 4,5 & 27,0 \\
\hline 9 & $107(14,5)$ & $97(8,4)$ & 9,4 & 19,6 \\
\hline 10 & $80(10,8)$ & $100(8,6)$ & 6,3 & 28,0 \\
\hline 11 & $90(12,2)$ & $97(8,4)$ & 12,2 & 17,5 \\
\hline 12 & $93(12,6)$ & $149(12,9)$ & 7,5 & 26,8 \\
\hline 13 & - & $100(8,6)$ & - & 26,0 \\
\hline 14 & - & $101(8,7)$ & - & 17,8 \\
\hline Total & 739 (100) & $1159(100)$ & 12,0 & 30,2 \\
\hline
\end{tabular}


Aos 5 anos de idade, os valores de ceod foram de 3,8 (IC95\%=2,9-4,7), em 1998, e de 2,4 (IC95\%=2,0-2,8) em 2004. Para os 12 anos de idade, a média do CPOD foi de 4,8 (IC95\%=4,0-5,6) em 1998 e de 2,7 (IC95\%=2,3-3,1) em 2004. Os componentes dos índices de cárie para a dentição decídua e permanente, em 1998 e 2004, pode ser visualizada na Figura 1.

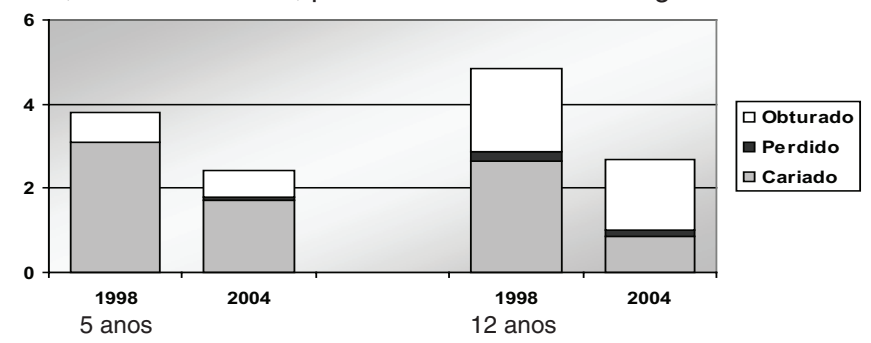

Figura 1. Experiência de cárie na dentição decídua (segundo os componentes, em média, do ceod) e permanente (componentes, em média, do CPOD) nos dois anos estudados. Rio das Pedras, 1998 e 2004.

A Figura 2 apresenta a distribuição dos escores do CPOD em cada uma das idades estudadas, tanto em 1998 quanto em 2004. No ano de 2004, também foram examinadas crianças de 13 e 14 anos de idade, dessa forma, o CPOD, aos 13 anos, foi de 3,6 $($ IC95\%=2,9-4,2), e aos 14 anos, foi de 4,3 (IC95\%=3,5-5,1).

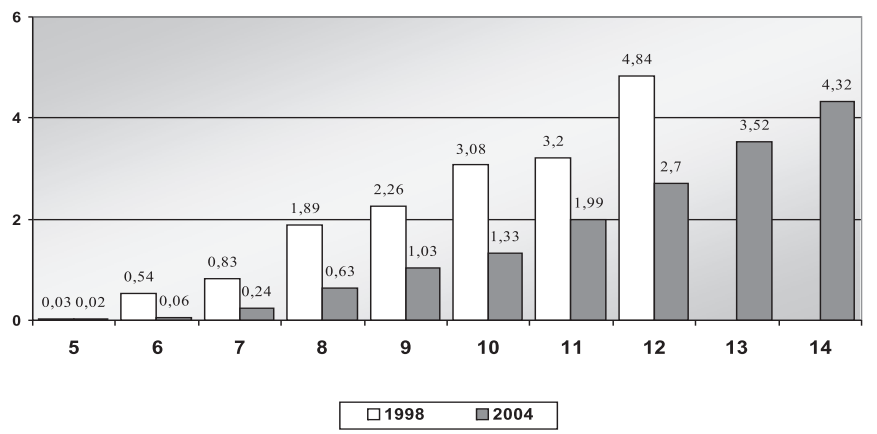

Figura 2. Média do índice CPOD segundo as idades estudadas. Rio das Pedras, 1998 e 2004.

O coeficiente de Gini, para a idade de 12 anos, foi de 0,41 em 1998, e de 0,53 em 2004. A distribuição gráfica do Coeficiente de
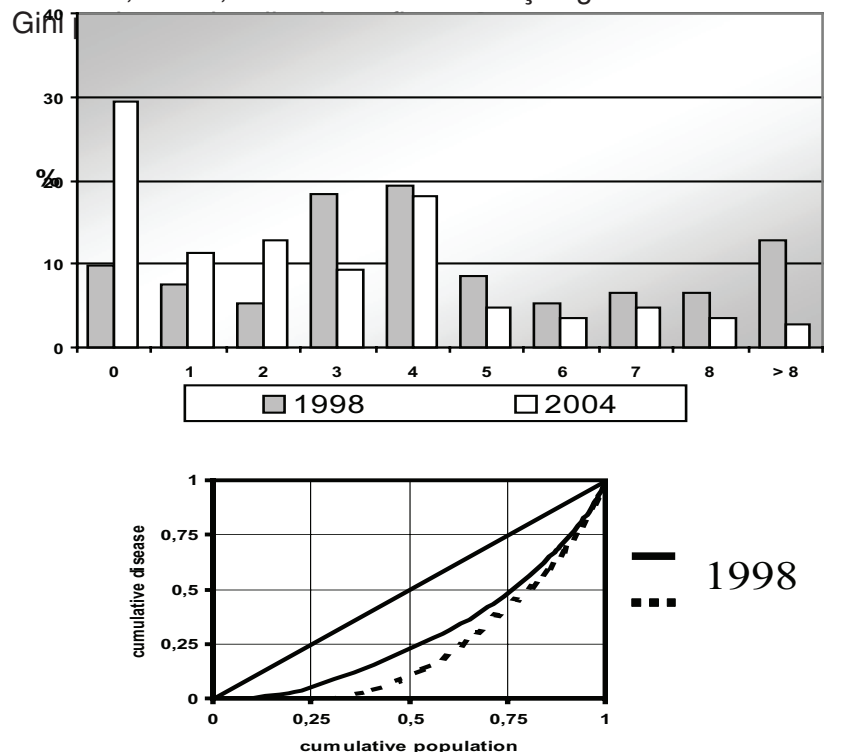

1998

Figura 3. CPOD e Coeficiente de Gini aos 12 anos de idade nos dois períodos estudados. Rio das Pedras, 1998-2004.
O índice $\mathrm{SiC}$, aos 12 anos de idade, foi de $9,03 \mathrm{em}$ 1998, e de 5,7 em 2004.

\section{Discussão}

Os resultados do presente trabalho mostraram que os valores médios de CPOD e ceod, no decorrer dos anos, diminuíram (Figura 1), assim como os valores percentuais que reduziram $43,75 \%$ para o CPOD aos 12 anos e $36,84 \%$ para o ceod aos 5 anos, no período estudado. Torna-se difícil explicar as causas desta redução em dois estudos transversais realizados em momentos distintos (1998 e 2004), entretanto possíveis fatores que podem contribuir para a ocorrência de tal fenômeno seriam a incorporação de fluoreto à água de abastecimento público introduzido a partir de 2001 no município de Rio das Pedras (Comunicação pessoal com Coordenador de Saúde Bucal do Município de Rio das Pedras, em 2004), além da melhoria das condições socioeconômicas da população (PERES et al., 2000; TRAEBERT et al., 2001; ANTUNES et al., 2002; BALDANI et al., 2002; CANGUSSU et al., 2002; TINANOFF et al., 2002) ou mesmo instituição de medidas preventivas pelos profissionais de saúde (RIHS et al., 2005).

Pôde-se verificar que nos dois levantamentos epidemiológicos realizados, o componente cariado foi o principal responsável pela diminuição do índice de cárie, tanto aos 5 anos quanto aos 12 anos de idade (Figura 1), sugerindo-se que mais dentes se mantiveram hígidos no período.

De acordo com a Figura 2, a média do CPOD aumentou em função da idade. Em 2004, houve um aumento do índice CPOD dos 12 aos 14 anos de idade, que se deve, provavelmente, ao irrompimento do segundo molar permanente. Tal fato mostra a importância de se considerar a inclusão de faixas etárias acima de 12 anos em levantamentos epidemiológicos futuros, como o grupo etário de 15 a 19 anos, grupo incluído no Projeto SB Brasil (BRASIL, 2003). Em estudo realizado por Gushi et al. (2005), condições de saúde bucal mais precárias foram verificadas em adolescentes de 15 a 19 anos, quando comparado ao índice CPOD encontrado na idade de 12 anos.

Uma das consequências observadas com o declínio da cárie dentária é a formação de grupos de polarização, em que uma pequena parcela da população concentra a maior parte das necessidades de tratamento decorrente da cárie (POWELL, 1998). Essa distribuição não uniforme da doença pode ser observada mesmo em países com baixa prevalência de cárie, em que os valores do CPOD são considerados muito baixos pela OMS (PITTS, 2002; PINE, 2003), onde ainda existem indivíduos com altos níveis da doença (BRATTHALL et al., 2000). Considerando os resultados deste estudo, pode-se observar que em contrapartida à melhora nos níveis de cárie dentária verificada pela redução do índice CPOD aos 12 anos em 2004, ainda existe uma pequena parcela desta população concentrando altos níveis da doença com índice SiC igual a 5,7.

Desta forma, não é possível avaliar esses indivíduos somente através de um único instrumento, ou seja, o índice CPOD, já que o mesmo apresenta-se como uma média, trazendo uma incompleta análise do impacto da experiência de cárie nos indivíduos mais afetados. Assim, esses altos escores são dissolvidos pela larga porção de indivíduos livres de cárie ou que possuem CPOD baixo (Figura 3).

Vários estudos vêm utilizando este índice e demonstrando altos valores do índice $\mathrm{SiC}$ em comparação com a média do CPOD da mesma população (NISHI, 2002; TAYANIN, 2002; WHELTON et al., 1997; PITTS, 2002).

Nos dois períodos estudados, apesar de ter diminuído a experiência de cárie nos índices ceod e CPOD, verificou-se que a desigualdade aumentou, ou seja, o coeficiente de Gini variou de 0,41 para 0,53 (Figura 3). Isto pode ser devido, em parte, ao próprio panorama histórico da cidade de Rio das Pedras que evidencia um perfil econômico voltado para o setor sucroalcooleiro, em que durante o período da safra da cana-de-açúcar indivíduos de outras regiões do Brasil migram para prestar serviço na colheita e assim, muitas vezes, 
acabam fixando residência, a fim de buscar melhores condições de vida. Desta forma, a população de migrantes se renova a cada período da safra da cana-de-açúcar, e assim, acaba contribuindo para esse quadro de aumento da desigualdade social com o reflexo na saúde bucal. Isto pode ser evidenciado quando se verifica que $12,8 \%$ dos examinados em 2004 não haviam nascido no município - dados não apresentados, obtidos durante a coleta dos dados.

Por fim, no período estudado, houve melhorias na saúde bucal desta população, entretanto, ainda existe uma parcela de indivíduos com altos níveis de cárie aos 12 anos. Além disso, é importante ressaltar que o período de 3 anos após o início da fluoretação (2001) ainda é relativamente curto para se atribuir somente a este método a redução da prevalência de cárie dentária no município, sugerindo a continuidade de estudos epidemiológicos voltados a indivíduos nascidos no município.

\section{Agradecimentos}

Às crianças e aos seus responsáveis, por autorizarem que os exames epidemiológicos fossem realizados. Ao coordenador de Saúde Bucal de Rio das Pedras, Fernando Nicolai, responsável pela coleta e disponibilização dos dados.

\section{Referências}

ANTUNES, J. L. F. et al. Spatial Analysis to Identify Differentials in Dental Needs by Area-based Measures. Community Dent. Oral Epidemiol., Copenhagen, v. 30, n. 2, p. 133-42, Apr. 2002.

BALDANI, M. S.; NARVAI, P. C.; ANTUNES, J. L. F. Cárie Dentária e Condições Sócio-econômicas no Estado do Paraná, Brasil, 1996. Cad. Saúde Pública, Rio de Janeiro, v.18, n. 3, p. 755-763, maio/jun. 2002.

BASTING, R. T.; PEREIRA, A. C.; MENEGHIM, M. C. Evaluation of Dental Caries Prevalence in Students from Piracicaba, SP, Brazil, After 25 Years of Fluoridation of the Public Water Supply. Rev. Odontol. Univ. São Paulo, São Paulo, v. 11, n. 4, p. 287-292, out./dez. 1997.

Ministério da Saúde. Levantamento Epidemiológico em Saúde Bucal: Brasil, zona urbana, 1986. Disponível em http://www. saude.gov.br/programas/bucal. Acesso em 24 de set. 2007.

BRATTHALL, D. Introducing the Significant Caries Index together with a Proposal for a New Global Oral Health Goal for 12-year-olds. Int. Dent. J., Bristol, v. 50, n. 6, p. 378-384, Dec. 2000.

CANGUSSU, M. C .T. et al. Cárie Dentária em Escolares de 12 e 15 Anos de Escolas Públicas e Privadas de Salvador, Bahia, Brasil, em 2001. Pesqui. Odontol. Bras. São Paulo, v. 16, n. 4, p. 379-384, dez. 2002.

CURY, J. A. et al. The Importance of Fluoride Dentifrices to the Current Dental Caries Prevalence in Brazil. Braz. Dent. J., Ribeirão Preto, v. 15, n. 3, p. 167-174, Sept./Dec. 2004.

CYPRIANO, S. et al. Saúde Bucal dos Pré-escolares, Piracicaba, Brasil, 1999. Rev. Saúde Pública, São Paulo, v. 37, n. 2, p. 247-253, abr. 2003.

FRIAS, A. C. et al. Custo da Fluoretação das Águas de Abastecimento Público, Estudo de Caso - Município de São Paulo, Brasil, Período de 1985-2003. Cad. de Saúde Pública, Rio de Janeiro, v. 22, n. 6, p. 1237-1246, jun. 2006.
GOMES, P. R. et al. Paulínia, São Paulo, Brasil: Situação da Cárie Dentária com Relação às Metas OMS 2000 e 2010. Cad. Saúde Pública, Rio de Janeiro, v. 20, n. 3, p. 866-870, maio/jun. 2004.

GUSHI, L. L. et al. Cárie Dentária em Adolescentes de 15 a 19 Anos de Idade no Estado de São Paulo, 2002. Cad. de Saúde Pública, Rio de Janeiro, v. 21, n. 5, p. 1383-1391, set./out. 2005.

HOFFMANN, R. H. S. et al. Experiência de Cárie Dentária em Crianças de Escolas Públicas e Privadas de um Município com Água Fluoretada. Cad. Saúde Pública, Rio de Janeiro, v. 20, n.2, p. 522-528, mar./abr. 2004.

MARTHALER, T. M. Changes in Dental Caries 1953-2003. Caries Res., Basel, v. 38, n . 3, p. 173-181, May/June 2004.

Ministério da Saúde. Projeto SB Brasil 2003: condições de saúde bucal da população brasileira 2002-2003. Brasília, 2003. Disponível em http://portalweb02.saude.gov.br/portal/arquivos/pdf/relatorio_brasil_sorridente.pdf. Acesso em 24 de set. 2007.

NADANOVSKY, P. Promoção da Saúde e a Prevenção das Doenças Bucais. In: PINTO, VG. Saúde Bucal Coletiva. São Paulo: Liv. Santos, p.139-222, 2000.

NARVAI, P. C.; FRAZÃO, P.; CASTELLANOS, R. A. Declínio da Experiência de Cárie em Dentes Permanentes de Escolares Brasileiros no Final do Século XX. Odont. Socied., São Paulo, v. 1, n. 1/2, p. 25-29, 1999.

NISHI, M. et al. Caries Experience of Some Countries and Areas Expressed by Significant Caries Index. Community Dent. Oral Epidemiol., Copenhagen, v. 30, n. 4, p. 296-301, Aug. 2002.

PERES, K. G. A.; BASTOS, J. R. M.; LATORRE, M. R. D. O. Relationship between Severity of Dental Caries and Social and Behavioral Factors in Children. Rev. Saúde Pública, São Paulo, v. 34, n. 4, p. 402-8, ago. 2000.

PIEPER, K.; SCHULTE, A. G. The Decline in Dental Caries among 12-year-old Children in Germany between 1994 and 2000. Community Dent. Health, London, v. 21, no. 3, p. 199-206, Sept. 2004.

PINE, C.; BURNSIDE, G.; CRAVEN, R. Inequalities in Dental Health in the North-West of England. Community Dent. Health, London, v. 20, n. 1, p. 55-56, Mar. 2003.

PITTS, N. B. et al. The Dental Caries Experience of 12-year-old Children in England and Wales. Surveys Coordinated by the British Association for the Study of Community Dentistry in 2000/2001. Community Dent. Health, London, v. 19, n. 1, p. 46-53, Mar. 2002.

POWELL, L. V. Factors Associated with Caries Incidence in an Elderly Population. Community Dent. Oral Epidemiol., Copenhagen, v. 26, n. 3, p. 170-176, June 1998.

RIHS, L. B. et al. Auto-aplicação de Gel Fluoretado por Pré-escolares de Piracicaba, SP. Rev. de Odontol. Univ. Cid. São Paulo, São Paulo, v.17, n.1, p. 55-62, jan./abr. 2005.

RIO DAS PEDRAS (SP). Prefeitura Municipal. Disponível em http://www.riodaspedras.sp.gov.br. Acesso em 24 de set. 2007.

SÃO PAULO (Estado). Secretaria da Saúde. Levantamento Epidemiológico em Saúde Bucal: Estado de São Paulo, 1998. São Paulo: SES-SP/Universidade de São Paulo, 1999. 
TAGLIAFERRO, E. P. S.; RIHS, L. B.; SOUZA, M. L. R. Caries Prevalence, Dental Fluorosis and Treatment Needs in Schoolchildren, Leme, SP, Brazil. Arq. Odontol., Belo Horizonte, v. 38, n. 3, p. 222-230, 2003.

TAYANIN, G. L.; RAMANATHAN, J.; BRATTHALL, D. Caries Prevalence and Some Caries Related Factors for 12 year old Children from Vientiane and Luang Prabang Provinces in Lao People's Democratic Republic. Odontostomastol. Trop., Dakar, v. 25, n. 98, p. 19-26, June 2002.

TINANOFF, N.; KANELLIS, M. J.; VARGAS, C. M. Current Understanding of the Epidemiology, Mechanisms and Prevention of Dental Caries in Preschool Children. Pediatr. Dent., Chicago, v. 24, n. 6 , p. 543-551, Nov./Dec. 2002.

TRAEBERT, J. L. et al. Prevalência e Severidade da Cárie Dentária em Escolares de Seis e Doze anos de Idade. Rev. Saúde Pública, São Paulo, v. 35, n. 3, p. 283-288, jun. 2001.

WEYNE, S. C. A Construção do Paradigma de Promoção de Saúde: um Desafio para as Novas Gerações. In: KRIGER, L. Promoção de Saúde Bucal. São Paulo: Artes Médicas, p. 1-26, 1997.

WHELTON, H.; O'MULLANE, D. M. Public Health Aspects of Oral Diseases and Disorders. In: PINE, C. M. (Ed.). Community Oral Health. Oxford: Wright, p.75-79, 1997.

WORLD HEALTH ORGANIZATION. Oral Health Surveys, Basics Methods. Geneva, 1997.

. Oral Health Surveys: Basic Methods. Geneva, 1987. 